\title{
Seroepidemiology of infection with Toxoplasma gondii in healthy blood donors of Durango, Mexico
} Cosme Alvarado-Esquivel*1, Miguel Francisco Mercado-Suarez², Alfredo Rodríguez-Briones ${ }^{3}$, Laura Fallad-Torres ${ }^{1}$, Julio Octavio Ayala-Ayala ${ }^{2}$, Luis Jorge Nevarez-Piedra ${ }^{3}$, Ehecatl Duran-Morales ${ }^{3}$, Sergio EstradaMartínez ${ }^{4}$, Oliver Liesenfeld ${ }^{5}$, José Ángel Márquez-Conde ${ }^{1}$ and Sergio Arturo Martínez-García ${ }^{1}$

\footnotetext{
Address: ${ }^{1}$ Faculty of Medicine, Juárez University of Durango State (UJED). Durango, Mexico, ${ }^{2}$ Mexican Institute of Social Insurance (IMSS), Durango, Mexico, ${ }^{3}$ State Center for Blood Transfusion, Secretary of Health. Durango, Mexico, ${ }^{4}$ Institute for Scientific Research, UJED. Durango, Mexico and 5 Institute for Microbiology and Hygiene, Campus Benjamin Franklin, Charité Medical School Berlin, Germany

Email: Cosme Alvarado-Esquivel* - alvaradocosme@yahoo.com; Miguel Francisco Mercado-Suarez - miguelfranms@hotmail.com; Alfredo Rodríguez-Briones - cetsdurango@yahoo.com.mx; Laura Fallad-Torres - lalis_f@hotmail.com; Julio Octavio AyalaAyala - miguelfranms@hotmail.com; Luis Jorge Nevarez-Piedra - cetsdurango@yahoo.com.mx; Ehecatl DuranMorales - cetsdurango@yahoo.com.mx; Sergio Estrada-Martínez - sem@mexico.com; Oliver Liesenfeld - oliver.liesenfeld@charite.de; José Ángel Márquez-Conde - jangelmc@hotmail.com; Sergio Arturo Martínez-García - sergiomicro@gmail.com

* Corresponding author
}

Published: 13 July 2007

BMC Infectious Diseases 2007, 7:75 doi:10.1 186/1471-2334-7-75
Received: 15 February 2007

Accepted: 13 July 2007

This article is available from: http://www.biomedcentral.com/I47/-2334/7/75

(c) 2007 Alvarado-Esquivel et al; licensee BioMed Central Ltd.

This is an Open Access article distributed under the terms of the Creative Commons Attribution License (http://creativecommons.org/licenses/by/2.0), which permits unrestricted use, distribution, and reproduction in any medium, provided the original work is properly cited.

\begin{abstract}
Background: Toxoplasma gondii (T. gondii) infection in blood donors could represent a risk for transmission in blood recipients. There is scarce information about the epidemiology of $T$. gondii infection in blood donors in Mexico. Therefore, we sought to determine the prevalence of $T$. gondii infection and associated sociodemographic and behavioral characteristics in a population of healthy blood donors of Durango City, Mexico.

Methods: Four hundred and thirty two blood donors in two public blood banks of Durango City, Mexico were examined for T. gondii infection between August to September 2006. Blood donors were tested for anti-T. gondii IgG and IgM antibodies by using enzyme-linked immunoassays (Diagnostic Automation Inc., Calabasas, CA, USA). Socio-demographic and behavioral characteristics from each participant were also obtained.

Results: Thirty two (7.4\%) of 432 blood donors had IgG anti-T. gondii antibodies. Eight (1.9\%) of them had also IgM anti-T. gondii antibodies. Multivariate analysis using logic regression showed that $T$. gondii infection was associated with the presence of cats at home (adjusted OR $=3.8 \mathrm{I} ; 95 \% \mathrm{Cl}$ : I.45-I0.0I). The age group of 45-60 years showed a significantly higher frequency of $T$. gondii infection than the group of $25-34$ years $(p=0.02)$. Blood donors without education had a significantly higher frequency of infection (15.8\%) than those with 13-19 years of education $(4.5 \%)(p=0.04)$. Other characteristics of blood donors including male gender, consumption of undercooked meat or blood transfusion did not show an association with infection.

Conclusion: The prevalence of $T$. gondii infection in healthy blood donors of Durango City, Mexico is lower than those reported in blood donors of south and central Mexico, and is one of the lowest reported in blood donors worldwide. $T$. gondii infection in our blood donors was most likely acquired by contact with cats. Prevalence of infection increased with age and decreased with educational level.
\end{abstract}




\section{Background}

Estimates indicate that up to one third of the world's population is infected by $T$. gondii $[1,2]$. Most infections in immunocompetent humans are asymptomatic and in up to $10 \%$ of infected individuals cervical lymphadenopathy or ocular disease occur [2]. Primary infection acquired during pregnancy could result in congenital toxoplasmosis $[2,3]$. In immunocompromised individuals, $T$. gondii infections could cause central nervous system disease as encephalitis or brain abscess [2,4]. Routes of parasite transmission in humans include 1) ingesting food or water that is contaminated with oocysts shed by cats; 2 ) eating undercooked or raw meat containing tissue cysts $[2,5,6]$ and; 3$)$ transplantation and blood transfusion [7$10]$.

The epidemiology of $T$. gondii infection in general, and in blood donors in particular has been poorly studied in Mexico. There is not any surveillance study or $T$. gondii screening program in blood donation, women in child bearing age or immunosuppressed patients. Studying a blood donor population is a valuable approach to determine epidemiological characteristics in adults of a community and might provide findings that could be found in the general adult population of the same community. There is a lack of information about the epidemiology of T. gondii infection in blood donors in northern Mexico. Therefore, we performed a cross-sectional study to determine the prevalence of $T$. gondii infection in blood donors of Durango City, Mexico and to identify characteristics of blood donors associated with seropositivity.

\section{Methods}

\section{Study design and study population}

We performed a cross sectional study (observational, prospective and descriptive survey) in the two largest blood banks of Durango City, Mexico. These blood banks were: blood bank 1, the General Hospital Blood Bank of the Mexican Institute of Social Insurance, and blood bank 2, the State Center for Blood Transfusion of the Secretary of Health. Inclusion criteria for the study subjects were: 1 ) voluntary blood donors; 2) aged 18 years and older; and 3 ) who accepted to participate in the study. All samples were routinely tested for antibodies against human immunodeficiency virus (HIV), hepatitis C virus (HCV), and Treponema pallidum, and hepatitis B virus surface antigen (HBsAg) in parallel to testing for antibodies against $T$. gondii. None of the blood donors were seropositive for HIV, HCV, HBsAg and Treponema pallidum. Two hundred and one blood donors of the first blood bank and 231 donors of the second blood bank attended from August to September 2006 were enrolled consecutively. In total 432 voluntary healthy blood donors participated in the study. Blood banks in Durango City are public, attend mostly low income blood donors, do not pay any blood dona- tion, and give donated blood or blood products in a free manner to hospitals.

\section{Ethical aspects}

This study was approved by the Institutional Ethical Committee. The purpose and procedures of the study were explained to all participants, and a written informed consent was obtained from all of them.

\section{Socio-demographic and behavioral data}

We used a standardized questionnaire to explore the characteristics of the blood donors. Socio-demographic data including age, birth place, residence place, marital status, occupation, educational level, and housing conditions index were obtained from all participants. Housing conditions index was obtained by using the Bronfman's criteria [11]. Briefly, five variables were evaluated: number of persons in the house, number of rooms in the house, material of the floor of the house, availability of drinkable water, and form of elimination of excreta. Contributing and confounding risk factors of behavioral data including blood transfusion or transplant history; animal contacts, cleaned up cat excrement, foreign travel, kind of meat consumption (pork, lamb, beef, goat, boar, chicken, turkey, rabbit, deer, squirrel, horse, fish and iguana), raw or undercooked meat consumption, unpasteurized milk or milk products consumption, untreated water consumption, dried or cured meat eaten (chorizo, ham, sausages or salami), unwashed raw vegetables or fruits consumption, contact with soil (gardening or agriculture), and eating outside of the home (at least once a year) from all blood donors were obtained.

\section{Laboratory tests}

Serum samples were obtained by centrifugation of fresh whole blood of the donors. The serum samples were transported from the blood banks to the Laboratory of Microbiology of the Faculty of Medicine (Juarez University of Durango State) where the samples were frozen down and kept stored at $-20^{\circ} \mathrm{C}$ until analyzed. Serum samples of blood donors were analyzed for anti-T. gondii IgG antibodies by a commercially available enzyme immunoassay "Toxoplasma IgG" kit (Diagnostic Automation Inc., Calabasas, CA, USA). In addition, sera positive for anti-T. gondii IgG antibodies were further analyzed for anti-T. gondii IgM antibodies by a commercially available enzyme immunoassay "Toxoplasma IgM" kit (Diagnostic Automation Inc., Calabasas, CA, USA). Both tests were performed in the Laboratory of Microbiology of the Faculty of Medicine following the instructions of the manufacturer. For both IgG test and IgM test, we calculated the Toxo $G$ index and Toxo $M$ index, respectively. These indexes are calculated for each determination by dividing the mean values of each sample by the cut-off calibrator mean value. A sample was considered positive for IgG or 
IgM when a Toxo $G$ index or a Toxo $M$ index was equal or greater than 1.0 (>8 IU/ml). A positive IgG test with a negative IgM test in a donor was interpreted as a chronic infection. A positive IgM test with a positive IgG test in a donor was interpreted as probability of recent infection.

\section{Statistical analysis}

The statistical analysis was performed with the aid of the software Epi Info version 3.3.2 and SPSS version 7.5 (SPSS Inc. Chicago, IL. USA). For calculation of the sample size we used a reference seroprevalence of $8.9 \%$ [12] as expected frequency of the factor under study, 10,000 as the size of population from which the sample was selected, a worst acceptable result of $12 \%$, and a confidence level of $95 \%$. The result of the calculation was 314 subjects. Descriptive statistics were used for numerical (mean and median) and categorical (frequency or percentage) variables. We used the Mantel-Haenszel test, and the Fisher exact test (when cells values were less than 5) for comparison of the frequencies among groups. For ordinal variables we used the $X^{2}$ for trend. Bivariate and multivariate analyses were used to assess the association between the characteristics of the subjects and T. gondii seropositivity. As a criterion for inclusion of variables in the multivariate analysis, we considered variables with a p value equal or less than 0.2 obtained in the bivariate analysis to allow potential confounding. Adjusted odd ratio (OR) and 95\% confidence interval (CI) were calculated by multivariate analysis using multiple, unconditional logistic regression. A p value less than 0.05 was considered statistically significant.

\section{Results}

Seroprevalence of anti-T. gondii antibodies

Seroprevalence of anti-T. gondii IgG antibodies in blood banks 1 and 2 were $9 \%(18 / 201)$ and 6.1\% (14/231), respectively. No statistically significant difference was found among the prevalences of anti-T. gondii IgG antibodies in each blood bank $(\mathrm{p}=0.25)$. Anti-T. gondii IgM antibodies were found in 2 of the 18 IgG positive donors from blood bank 1, and in 6 of the 14 IgG positive donors from blood bank 2. Overall, thirty two (7.4\%) of 432 blood donors were positive for anti-T. gondii IgG antibodies and eight (1.9\%) of them were also positive for anti-T. gondii IgM antibodies.

\section{Socio-demographic characteristics and other possible risk factors associated with seropositivity}

General socio-demographic characteristics of the 432 blood donors studied are shown in Table 1 . The mean age of the blood donors was $30.4+/-8.8$ years (median: 29 years; range: 18 to 60 years). The majority of blood donors were born in Mexico and resided in urban areas of Durango State. Most blood donors were males, have studied up to 12 years, and used to live in regular or good housing conditions. The highest frequency of infection (11\%) was found in the age group of 35-60 years while the lowest frequency $(4.3 \%)$ in the age group of $25-34$ years. The difference among these frequencies (11\% vs $4.3 \%)$ was statistically significant $(\mathrm{p}=0.02)$. There was a trend of higher seroprevalence of anti-T. gondii IgG antibodies as educational level decreased ( $\mathrm{p}=0.04)$. Blood donors without education had a significantly higher frequency of infection (15.8\%) than those with $13-19$ years of education $(4.5 \%)(\mathrm{p}=0.04)$.

In the bivariate analysis, six variables were identified as possible risk factors associated with $T$. gondii infection: 1 ) cats at home $(\mathrm{p}=0.01) ; 2)$ cats in the neighborhood $(\mathrm{p}=$ $0.19)$; and 3 ) living in a house with soil floors $(\mathrm{p}=0.19)$. In addition, we found two variables that showed possible negative association with $T$. gondii: 1 ) pork meat consumption $(\mathrm{p}=0.05) ; 2)$ turkey meat consumption $(\mathrm{p}=$ $0.01)$; and 3 ) sausage consumption $(\mathrm{p}=0.2)$. Other sociodemographic and behavioral characteristics of the blood donors did not show any possible association with T. gondii infection. Table 2 shows the results of the bivariate analysis of selected variables and the results of T. gondii seropositivity. Further analysis by using logistic regression revealed that only the variable cats at home was significantly associated with $T$. gondii seropositivity (adjusted $\mathrm{OR}=3.81 ; 95 \% \mathrm{CI}:$ 1.45-10.01) (Table 3).

\section{Discussion}

In the present study, we found a $7.4 \%$ seroprevalence of $T$. gondii infection in blood donors of Durango, Mexico. This prevalence could be considered low and questions on the sensitivity of the test might rise. However, we think that the sensitivity of the tests was very good and the results were reliable. The sensitivity of the IgG test is 94\%. Quality control of both IgG and IgM test runs showed valid results. The low prevalence of seropositivity for $T$. gondii found is comparable with that detected in other healthy population of Durango City using a different detection method [13]. This prevalence is much lower than that reported in the southern Mexican State of Yucatan [14], and the central Mexican State of Jalisco [15] where researchers found that $69 \%$ and $29 \%$ of blood donors were positive for anti-T. gondii antibodies, respectively. Our prevalence found in Durango City is also much lower than those reported in blood donors from other Latin American countries, as Brazil [16], Cuba [17], and Chile [18]. Similarly, our prevalence is much lower than that found in blood donors in Malaysia [19], Saudi Arabia [20], Czech Republic [21], and Mali [22] were prevalences varied from $21 \%$ to $52.1 \%$. In contrast, our prevalence was comparable with the $9 \%$ prevalence found in blood donors of Loei Province, Thailand [23]. It is possible that differences in the characteristics of the blood donors and differences in the environments might contribute to 
Table I: Socio-demographic characteristics of the $\mathbf{4 3 2}$ blood donors in Durango, Mexico.

\begin{tabular}{|c|c|c|c|c|}
\hline \multirow[b]{2}{*}{ Characteristic } & \multicolumn{2}{|c|}{ Donors studied ${ }^{a}$} & \multicolumn{2}{|c|}{ Donors positive for anti-T. gondii antibodies } \\
\hline & No. & $\%$ & No. & $\%$ \\
\hline \multicolumn{5}{|l|}{ Gender } \\
\hline Male & 374 & 86.6 & 28 & 7.5 \\
\hline Female & 58 & 13.4 & 4 & 6.9 \\
\hline \multicolumn{5}{|l|}{ Age groups (years) } \\
\hline $18-24$ & 135 & 31.3 & 10 & 7.4 \\
\hline $25-34$ & 161 & 37.3 & 7 & 4.3 \\
\hline $35-60$ & 136 & 31.4 & 15 & II \\
\hline \multicolumn{5}{|l|}{ Born in Mexico } \\
\hline Yes & 427 & 99.8 & 31 & 7.3 \\
\hline No & 1 & 0.2 & 0 & 0 \\
\hline \multicolumn{5}{|l|}{ Residence Place } \\
\hline Durango State & 424 & 98.1 & 31 & 7.3 \\
\hline Other Mexican State & 7 & 1.6 & 0 & 0 \\
\hline Abroad & 1 & 0.2 & 0 & 0 \\
\hline \multicolumn{5}{|l|}{ Residence area } \\
\hline Urban & 304 & 71.5 & 20 & 6.6 \\
\hline Suburban & 45 & 10.6 & 6 & 13.3 \\
\hline Rural & 76 & 17.9 & 5 & 6.6 \\
\hline \multicolumn{5}{|l|}{ Educational level } \\
\hline No education & 38 & 9 & 6 & $15.8^{e}$ \\
\hline Up to 12 years (College) & 295 & 69.7 & 21 & 7.1 \\
\hline 13-19 years (Undergraduate) & 88 & 20.8 & 4 & 4.5 \\
\hline Graduate & 2 & 0.5 & 0 & 0 \\
\hline \multicolumn{5}{|l|}{ Occupation } \\
\hline No laborerb & 207 & 48.3 & 14 & 6.8 \\
\hline Laborerc & 143 & 33.3 & 12 & 8.4 \\
\hline Other/unemployed d & 79 & 18.4 & 5 & 6.3 \\
\hline \multicolumn{5}{|l|}{ Housing conditions index } \\
\hline Good & 215 & 62.3 & 16 & 7.4 \\
\hline Regular & 95 & 27.5 & 10 & 10.5 \\
\hline Bad & 35 & 10.1 & 0 & 0 \\
\hline
\end{tabular}

aBlood donors with available data.

bNon laborer = Professional, businessman, employee.

cLaborer = Farmer and worker in factory or construction areas.

dOther/unemployed $=$ student and housewife.

eStatistically significant difference among the educational level groups by $X^{2}$ for trend: $p=0.04$.

explain the lower prevalence of $T$. gondii infection found in our blood donor population than those reported in blood donors from southern and central Mexico or other countries. Most of our blood donors belonged to a low socio-economic level and eating meat is not a frequent practice among them. In addition, consumption of under- cooked or raw meat is rarely found among our blood donors. Environmental characteristics of Durango City as a dry climate, and a high altitude may also contribute to explain the low frequency of infection. This explanation is supported by previous observations that prevalence of $T$. gondii infection in populations living in dry climates was

Table 3: Multivariate analysis of selected characteristics of the $\mathbf{4 3 2}$ blood donors and their association with $T$. gondii infection.

\begin{tabular}{lccc}
\hline Characteristic $^{\mathrm{a}}$ & Adjusted odds ratio & 95\% Confidence Interval & P value \\
\hline Pork meat consumption & 0.38 & $0.11-1.28$ & 0.12 \\
Turkey meat consumption & 0.34 & $0.09-1.23$ & 0.1 \\
Cats at home & 3.81 & $1.45-10.01$ & 0.006 \\
Cats in the neighborhood & 0.64 & $0.25-1.67$ & 0.36 \\
House with soil floors & 2.94 & $0.26-32.8$ & 0.38 \\
Sausage consumption & 0.7 & $0.22-2.26$ & 0.55
\end{tabular}

aThe variables included were those with a $p \leq 0.20$ obtained in the bivariate analysis.

bAdjusted by age, housing conditions index, and the rest of characteristics included in this table. 
Table 2: Bivariate analysis of putative risk factors for infection with T. gondii in the $\mathbf{4 3 2}$ blood donors from Durango, Mexico.

\begin{tabular}{|c|c|c|c|}
\hline Characteristic & Blood donors ${ }^{\mathrm{a}}$ No. (\%) & Positive test for anti-T gondii antibodies No. (\%) & $P$ value \\
\hline \multicolumn{4}{|l|}{ Pork meat consumption } \\
\hline Yes & $361(84.9)$ & $21(5.8)$ & 0.05 \\
\hline No & $64(15.1)$ & $8(12.5)$ & \\
\hline \multicolumn{4}{|l|}{ Turkey meat consumption } \\
\hline Yes & $126(29.5)$ & $3(2.4)$ & 0.01 \\
\hline No & $301(70.5)$ & $29(9.6)$ & \\
\hline \multicolumn{4}{|l|}{ Sausage consumption } \\
\hline Yes & $367(85.7)$ & $25(6.8)$ & 0.2 \\
\hline No & $61(14.3)$ & 7 (II.5) & \\
\hline \multicolumn{4}{|l|}{ Degree of meat cooking } \\
\hline Raw or undercooked & $10(2.3)$ & I (I0.0) & 0.53 \\
\hline Well done & $422(97.7)$ & $31(7.3)$ & \\
\hline \multicolumn{4}{|c|}{ Unwashed raw vegetable or fruit consumption } \\
\hline Yes & $59(13.8)$ & $3(5.1)$ & 0.59 \\
\hline No & $367(86.2)$ & $29(7.9)$ & \\
\hline \multicolumn{4}{|l|}{ Cats at home } \\
\hline Yes & $116(27)$ & $15(12.9)$ & 0.01 \\
\hline No & $314(73)$ & $17(5.4)$ & \\
\hline \multicolumn{4}{|l|}{ Cleaning cat feces } \\
\hline Yes & $46(11.1)$ & $4(8.7)$ & 0.46 \\
\hline No & $367(88.9)$ & $27(7.4)$ & \\
\hline \multicolumn{4}{|l|}{ Cats in the neighborhood } \\
\hline Yes & $155(36.2)$ & $15(9.7)$ & 0.19 \\
\hline No & $273(63.8)$ & $17(6.2)$ & \\
\hline \multicolumn{4}{|l|}{ Gardening or agriculture } \\
\hline Yes & $123(29.4)$ & $7(5.7)$ & 0.45 \\
\hline No & $296(70.6)$ & $23(7.8)$ & \\
\hline \multicolumn{4}{|l|}{ Blood transfusion } \\
\hline Yes & $6(1.4)$ & $0(0)$ & 0.49 \\
\hline No & $419(98.6)$ & $30(7.2)$ & \\
\hline \multicolumn{4}{|l|}{ Soil floors } \\
\hline Yes & II (2.8) & $2(18.2)$ & 0.19 \\
\hline No & $389(97.3)$ & $28(7.2)$ & \\
\hline
\end{tabular}

aBlood donors with available data.

lower than those living in other climates $[2,24,25]$, and lower in populations living in high altitudes than those in low altitudes either in humans $[26,27]$ or in animals [28].

With respect to the demographic characteristics of the blood donors, we observed that the frequency of seropositivity increased with age, and this observation agrees with those reported in other studies $[16,20]$. In addition, we observed that seropositivity to $T$. gondii decreased as educational level in donors increased $(\mathrm{p}=0.04)$. To the best of our knowledge, a similar observation in blood donors had not been reported. This finding deserves further study in which additional results might confirm or challenge our finding. We can not state that water influenced our result since untreated water consumption or other water variables were not associated with seropositivity in our study. Socio-economic status does not explain our finding since most donors belonged to a low income population. We speculate that high education is linked to good hygienic sanitary practices thereby reducing the transmission of the parasite. Male and female blood donors showed comparable prevalences of $T$. gondii infection, and this result does not support previous observations that showed a higher prevalence of infection in male than female blood donors $[16,23]$. The number of female donors in this study was much lower than that of male donors. Therefore, further studies are needed to elucidate risk factors associated with seropositivity in female donors. The knowledge of factors associated with seroconversion in women is particularly important during their reproductive age in order to design preventive measures and avoid acute infections during pregnancy. The fact that in this study the variable "cats at home" was associated with infection indicates that $T$. gondii infection in our infected population might have occurred by ingesting parasite oocysts in contaminated food or water. Contact with cats has not always been associated with $T$. gondii seropositivity in epidemiology studies, as shown in a previous study in blood donors and HIV patients [19] or in pregnant women [13]. In contrast, in our study we did not 
observe an association of seropositivity with consumption of any meat explored indicating that meat consumption was not relevant in parasite transmission in the blood donors studied. Meat consumption has been found to be an important factor in parasite transmission in several studies $[13,29]$. However, in some studies no association between $T$. gondii infection and meat consumption has been found $[12,19]$. The very low number of donors with anti-T. gondii antibodies found in this study reduces the statistical power to find further associations between seropositivity and the epidemiological characteristics in blood donors. Certainly by increasing the sample size the statistical power increases and some risk factors with borderline significance might turn out to become significant. This is especially interesting for risk factors including meat consumption, [13,29], and soil floors [13].

Interestingly, nearly $2 \%$ of our blood donors had also IgM antibodies against $T$. gondii. The absence of this infection marker in subjects with anti-T. gondii IgG antibodies indicates a chronic infection but its presence does not necessarily indicate an acute infection. We performed detection of IgM antibodies only in IgG positive samples because the presence of IgM antibodies alone is rarely seen. AntiT. gondii IgG antibodies appears very early after infection [30], therefore, the window period between the appearance of IgM and the appearance of IgG is extremely short and the probability to find an IgM positive/IgG negative infected subject seems to be quite low to financially justify a systematic IgM screening in a cross sectional study. In addition, seropositivity to IgM alone is not considered an acceptable diagnostic criterion for acute infection. Anti-T. gondii-specific IgM antibodies are detectable early after infection and can persist for prolonged times after infection [2,31]. IgM-positive donors with parasitemia may hold a potential for parasite transmission by blood transfusion. We were unable to judge whether a fraction of our blood donors might represent a risk group for parasite transmission by blood transfusion as reported previously $[8,15]$.

\section{Conclusion}

We concluded that the prevalence of $T$. gondii infection in blood donors of Durango City, Mexico is low as compared with those reported in central and south Mexico and the majority of other countries. T. gondii infection in our blood donors was most likely acquired by contact with cats. Infection increased with age and decreased with educational level.

\section{Competing interests}

The author(s) declare that they have no competing interests.

\section{Authors' contributions}

CAE conceived and designed the study protocol, participated in the coordination and management of the study, performed the laboratory tests and data analysis, and wrote the manuscript. MFMS, ARB, JOAA, LJNP, EDM, and JAMC applied the questionnaires and performed the data analysis. SEM performed the statistical analysis. LFT performed the laboratory tests. OL designed the study protocol, performed the data analysis, and wrote the manuscript. SAMG performed the data analysis.

\section{References}

I. Hill DE, Chirukandoth S, Dubey JP: Biology and epidemiology of Toxoplasma gondii in man and animals. Anim Health Res Rev 2005, 6:4I-6I.

2. Montoya JG, Liesenfeld O: Toxoplasmosis. Lancet 2004, 363:1965-1976.

3. Kravetz JD, Federman DG: Toxoplasmosis in pregnancy. Am J Med 2005, I I 8:212-2।6.

4. Walker M, Zunt JR: Parasitic central nervous system infections in immunocompromised hosts. Clin Infect Dis 2005, 40: $1005-1015$.

5. Dubey JP: Toxoplasmosis - a waterborne zoonosis. Vet Parasitol 2004, I 26:57-72.

6. Dawson D: Foodborne protozoan parasites. Int J Food Microbiol 2005, 103:207-227.

7. Barsoum RS: Parasitic infections in transplant recipients. Nat Clin Pract Nephrol 2006, 2:490-503.

8. Figueroa Damian R: Risk of transmission of infectious diseases by transfusion. Ginecol Obstet Mex 1998, 66:277-283.

9. Wurzner R: Transplantation-associated infections. Verh Dtsch Ges Pathol 2004, 88:85-88.

10. Campbell AL, Goldberg CL, Magid MS, Gondolesi G, Rumbo C, Herold BC: First case of toxoplasmosis following small bowel transplantation and systematic review of tissue-invasive toxoplasmosis following noncardiac solid organ transplantation. Transplantation 2006, 81:408-4I7.

II. Bronfman M, Guiscafré H, Castro V, Castro R, Gutiérrez G: La medición de la desigualdad: una estrategia metodológica, análisis de las características socioeconómicas de la muestra. Arch Invest Med 1988, 19:351-360.

12. Alvarado-Esquivel C, Alanis-Quinones OP, Arreola-Valenzuela MA, Rodriguez-Briones A, Piedra-Nevarez LJ, Duran-Morales E, EstradaMartinez S, Martinez-Garcia SA, Liesenfeld O: Seroepidemiology of Toxoplasma gondii infection in psychiatric inpatients in a northern Mexican city. BMC Infect Dis 2006, 19:178.

13. Alvarado-Esquivel C, Sifuentes-Alvarez A, Narro-Duarte SG, EstradaMartinez S, Diaz-Garcia JH, Liesenfeld O, Martinez-Garcia SA, Canales-Molina A: Seroepidemiology of Toxoplasma gondii infection in pregnant women in a public hospital in northern Mexico. BMC Infect Dis 2006, 6:1 I3.

14. Gongora-Biachi RA, Gonzalez-Martinez P, Castro-Sansores C, Alvarez-Moguel R, Pavia-Ruz N, Lara-Perera D, Alonzo-Salomon G, Palacios-Perez E: Antibodies against Toxoplasma gondii in patients with HIV in Yucatán. Rev Invest Clin 1998, 50:419-422.

15. Galvan Ramirez ML, Covarrubias X, Rodriguez R, Troyo R, Alfaro N, Correa D: Toxoplasma gondii antibodies in Mexican blood donors. Transfusion 2005, 45:28I-282.

16. Coelho RA, Kobayashi M, Carvalho LB Jr: Prevalence of IgG antibodies specific to Toxoplasma gondii among blood donors in Recife, Northeast Brazil. Rev Inst Med Trop Sao Paulo 2003, 45:229-231.

17. Martín-Hernández I, García-Izquierdo SM: Prevalencia de anticuerpos IgG contra Toxoplasma gondii en donantes de sangre cubanos. Rev Biomed 2003, 14:247-25I.

18. Zamorano CG, Contreras MC, Villalobos S, Sandoval L, Salinas P: Seroepidemiological survey of human toxoplasmosis in Osorno, Region X, Chile, 1998. Bol Chil Parasitol 1999, 54:33-36.

19. Nissapatorn V, Kamarulzaman A, Init I, Tan LH, Rohela M, Norliza A, Chan LL, Latt HM, Anuar AK, Quek KF: Seroepidemiology of toxoplasmosis among HIV-infected patients and healthy blood donors. Med J Malaysia 2002, 57:304-310. 
20. Al-Amari OM: Prevalence of antibodies to Toxoplasma gondii among blood donors in Abha, Asir Region, south-western Saudi Arabia. J Egypt Public Health Assoc 1994, 69:77-88.

21. Svobodova V, Literak I: Prevalence of IgM and IgG antibodies to Toxoplasma gondii in blood donors in the Czech Republic. Eur J Epidemiol 1998, I4:803-805.

22. Maiga I, Kiemtore P, Tounkara A: Prevalence of antitoxoplasma antibodies in patients with acquired immunodeficiency syndrome and blood donors in Bamako. Bull Soc Pathol Exot 200I, 94:268-270.

23. Pinlaor S, leamviteevanich K, Pinlaor P, Maleewong W, Pipitgool V: Seroprevalence of specific total immunoglobulin (Ig), IgG and IgM antibodies to Toxoplasma gondii in blood donors from Loei Province, Northeast Thailand. Southeast Asian J Trop Med Public Health 2000, 3 I : I23-I 27.

24. Jenum PA, Kapperud G, Stray-Pedersen B, Melby KK, Eskild A, Eng J: Prevalence of Toxoplasma gondii specific immunoglobulin $\mathbf{G}$ antibodies among pregnant women in Norway. Epidemiol Infect 1998, 120:87-92.

25. Velasco-Castrejon O, Salvatierra-Izaba B, Valdespino JL, Sedano-Lara AM, Galindo-Virgen S, Magos C, Llausas A, Tapia-Conyer R, Gutierrez G, Sepulveda J: Seroepidemiology of toxoplasmosis in Mexico. Salud Publica Mex 1992, 34:222-229.

26. Hershey DW, McGregor JA: Low prevalence of Toxoplasma infection in a Rocky Mountain prenatal population. Obstet Gynecol 1987, 70:900-902

27. Rai SK, Shibata H, Sumi K, Kubota K, Hirai K, Matsuoka A, Kubo T, Tamura T, Basnet SR, Shrestha HG, Mahajan RC: Seroepidemiological study of toxoplasmosis in two different geographical areas in Nepal. Southeast Asian J Trop Med Public Health 1994, 25:479-484.

28. Rajkhowa S, Sarma DK, Rajkhowa C: Seroprevalence of Toxoplasma gondii antibodies in captive mithuns (Bos frontalis) from India. Vet Parasitol 2006, 135:369-374.

29. Cook AJ, Gilbert RE, Buffolano W, Zufferey J, Petersen E, Jenum PA, Foulon W, Semprini AE, Dunn DT: Sources of Toxoplasma infection in pregnant women: European multicentre case-control study. European Research Network on Congenital Toxoplasmosis. BMJ 2000, 32 I: | 42-147.

30. Pfrepper KI, Enders G, Gohl M, Krczal D, Hlobil H, Wassenberg D, Soutschek E: Seroreactivity to and avidity for recombinant antigens in toxoplasmosis. Clin Diagn Lab Immunol 2005, 12:977-982

31. Liesenfeld O, Press C, Montoya JG, Gill R, Isaac-Renton JL, Hedman $\mathrm{K}$, Remington JS: False-positive results in immunoglobulin $M$ (IgM) Toxoplasma antibody tests and importance of confirmatory testing: the Platelia Toxo IgM test. J Clin Microbiol I997, 35:174- 178 .

\section{Pre-publication history}

The pre-publication history for this paper can be accessed here:

http://www.biomedcentral.com/1471-2334/7/75/prepub

\section{Publish with Bio Med Central and every scientist can read your work free of charge}

"BioMed Central will be the most significant development for disseminating the results of biomedical research in our lifetime. "

Sir Paul Nurse, Cancer Research UK

Your research papers will be:

- available free of charge to the entire biomedical community

- peer reviewed and published immediately upon acceptance

- cited in PubMed and archived on PubMed Central

- yours - you keep the copyright
BioMedcentral 